\title{
IAEA Nuclear Databases for Applications
}

\author{
Otto Schwerer \\ Nuclear Data Section, International Atomic Energy Agency, Vienna, Austria
}

Received on 30 October, 2002

\begin{abstract}
The Nuclear Data Section (NDS) of the International Atomic Energy Agency (IAEA) provides nuclear data services to scientists on a worldwide scale with particular emphasis on developing countries. More than 100 data libraries are made available cost-free by Internet, CD-ROM and other media. These databases are used for practically all areas of nuclear applications as well as basic research. An overview is given of the most important nuclear reaction and nuclear structure databases, such as EXFOR, CINDA, ENDF, NSR, ENSDF, NUDAT, and of selected special purpose libraries such as FENDL, RIPL, RNAL, the IAEA Photonuclear Data Library, and the IAEA charged-particle cross section database for medical radioisotope production. The NDS also coordinates two international nuclear data centre networks and is involved in data development activities (to create new or improve existing data libraries when the available data are inadequate) and in technology transfer to developing countries, e.g. through the installation and support of the mirror web site of the IAEA Nuclear Data Services at IPEN (operational since March 2000) and by organizing nuclear-data related workshops. By encouraging their participation in IAEA Co-ordinated Research Projects and also by compiling their experimental results in databases such as EXFOR, the NDS helps to make developing countries' contributions to nuclear science visible and conveniently available. The web address of the IAEA Nuclear Data Services is http://wwwnds.iaea.org and the NDS mirror service at IPEN (Brasil) can be accessed at http://www-nds.ipen.br/
\end{abstract}

\section{Nuclear data types and data centers}

\section{I.1 What are nuclear data?}

Nuclear data are quantitative results of scientific investigations of the nuclear properties of matter. They describe properties of atomic nuclei and the fundamental physical relationships governing their interactions, thereby characterizing the physical processes underlying all nuclear technologies. Examples of nuclear data include cross sections, halflives, decay modes and decay radiation properties, and $\gamma$ rays from radionuclides. The scope of the data collections includes all 85 natural elements with 290 stable isotopes and more than 2500 radionuclides.

Applications of nuclear data include all areas of nuclear science and technology, covering energy applications (fission reactor design; nuclear fuel cycles; nuclear safety; reactor monitoring and fluence determination; waste disposal and transmutation; accelerator driven systems; fusion device design and plasma processing technologies) as well as non-energy applications (cancer radiotherapy; production of radioisotopes for medical and industrial applications; personnel dosimetry and radiation safety; nuclear safeguards; environmental monitoring and clean-up; materials analysis and process control; radiation damage studies; detection of concealed explosives and illegal drugs; exploration for oil and other minerals) and basic research (e.g. nuclear astro- physics) and education. A recent review of nuclear data for applications can be found in Ref.[1].

\section{I.2 The role of the IAEA Nuclear Data Section}

The Nuclear Data Section (NDS) of the International Atomic Energy Agency (IAEA) carries out IAEA activities concerning the development and dissemination of nuclear and atomic data for applications. In addition, the NDS is involved in technology transfer activities to assist scientists of developing countries within this area of expertise.

Main tasks of the data center include compilation of new data in the databases EXFOR and CINDA (in cooperation with other participating data centers, see below), collection and maintenance of general-purpose and specialized evaluated data libraries, online and off-line nuclear data services with particular emphasis on the needs of developing countries, and co-ordination of nuclear data center networks.

Nuclear data development is conducted mainly through Co-ordinated Research Projects (CRPs). In such projects, which usually result in the production of a new (or significant upgrade of an existing) database, typically 4-10 scientific groups from different countries work together under IAEA contracts or agreements over a period of 3-4 years. Examples of recent CRPs run by the NDS are given in Table 1. 


\begin{tabular}{|l|l|c|}
\hline Topic & Duration & Participants \\
\hline \hline Fission Yield Data $(\leq 20 \mathrm{MeV})$ & $1991-96$ & 7 \\
\hline Photon Production Data & $1994-98$ & 9 \\
\hline Medical Radioisotope Production & $1995-99$ & 7 \\
\hline Photonuclear Data & $1996-2000$ & 7 \\
\hline Fission Yield Data for Transmutation $(\leq 150 \mathrm{MeV})$ & $1997-2002$ & 10 \\
\hline X- and Gamma-Ray Standards & $1998-2002$ & 8 \\
\hline Input Parameter Testing (RIPL-II) & $1998-2002$ & 8 \\
\hline Prompt Gamma Activation Analysis (PGAA) & $1999-2003$ & 5 \\
\hline Nuclear data for Th-U fuel cycle & approved & \\
\hline Standard Cross Sections for Light Elements & approved & \\
\hline Nuclear Data Evaluation for Emerging Technologies (RIPL-III) & planned & \\
\hline Nuclear Data for Production of Therapeutic Radionuclides & planned & \\
\hline
\end{tabular}

Table 1. Recent nuclear data related Co-ordinated Research Projects

Technology transfer to developing countries is carried out in several ways: through Technical Co-operation projects, such as the recent "Latin American Mirror Server Project" providing online nuclear data service to Latin American countries with insufficient Internet connection to the NDS, by installing a mirror server in Brazil, or the installation of a "mini data center" on a workstation in Ghana. In addition, nuclear data workshops are organized on a regular basis, usually held at the Abdus Salam International Centre for Theoretical Physics in Trieste, Italy. Topics include "Nuclear Reaction Data and Nuclear Reactors: Physics, Design and Safety" (even years) and "Nuclear Data for Science and Technology" (odd years, with changing special topics such as medical physics, accelerator driven waste incineration, and materials analysis).

The Atomic and Molecular Data Unit of the NDS keeps databases for fusion energy, plasma research and other applications, such as ALADDIN (numerical data) and AMBDAS (bibliographic data), and issues specialized publications (e.g. CIAMDA). The databases are kept on a separate server [2]. The remainder of this lecture deals only with the nuclear data services of NDS.

\section{I.3 Nuclear data types}

Nuclear data are commonly categorized in two main groups: nuclear reaction data, describing the interactions of various projectiles such as neutrons, protons or photons with target nuclei, and nuclear structure and decay data, describing nuclear levels, half-lives and radioactive decay radiations. For both groups, the type of information given can be experimental data or evaluated data (both numeric) or bibliographic.

- Bibliographic data: References with some description of the contents, but no numerical data. Examples are CINDA (Computer Index of Neutron Data) and NSR (Nuclear Science References).

- Experimental data: Results of individual measurements as reported by the authors. The most important example is EXFOR/CSISRS, the library for experimental nuclear reaction data.
- Evaluated data libraries contain recommended data based on all data available from experiments and/or theory, arrived at after critical analysis of experimental data and their uncertainties, inter- and extrapolation, and/or nuclear model calculations. They are stored in strictly defined formats such as ENDF-6 (the international format for evaluated nuclear reaction data) or ENSDF (the format of the Evaluated Nuclear Structure Data File). The main cross section libraries in ENDF format usually contain also the relevant decay data needed in the main applications.

- Nuclear reaction data include cross sections, angular and energy distributions of secondary particles, resonance parameters, and related quantities. For neutroninduced reactions up to $20 \mathrm{MeV}$, the libraries are rather complete; the coverage for higher energies is less complete but improving. Experimental data are found in EXFOR, the related bibliography in CINDA; several evaluated data libraries exist up to $20 \mathrm{MeV}$ or higher. For charged-particle induced and photonuclear reactions, selected experimental data are compiled in EXFOR and few evaluations exist. Heavy-ion data are partly compiled in EXFOR.

- Nuclear structure and decay data: half-lives, decay schemes, nuclear level properties, energies and intensities of $\gamma$-rays and emitted particles, atomic masses. The major database is ENSDF, while related bibliographic data are contained in NSR. There are many other nuclear structure and decay data libraries, mostly derived from or related to ENSDF; some of these are listed in Section 2 below, others - not available from NDS - include the Table of Isotopes [3], the Isotope Explorer [4], a computer program for viewing ENSDF and for interactive access to nuclear structure and decay data, and NUBASE [5], a library of nuclear and decay properties.

\section{I.4 Nuclear data center networks}

Both the collection and the distribution of nuclear data are organised on a world-wide scale. Two international networks are coordinated by the IAEA: the Network of Nuclear 
Reaction Data Centers [6] and the Nuclear Structure and Decay Data Network [7] (see Table 2). The data centers participating in these networks are involved in the various stages of data preparation between measurement and application (i.e. compilation, review and/or evaluation, processing, distribution).

Specialized data centers cooperate with the major cen- ters in the various data center functions (in particular data compilation and evaluation). The sharing of work on a world-wide basis in the various areas of work, including data distribution, is defined partly geographically and partly by data types (scope), and is coordinated by the IAEA Nuclear Data Section.

\begin{tabular}{|l|l|}
\hline Nuclear Reaction Data Centers Network & Nuclear Structure Data Centers Network \\
\hline \hline $\begin{array}{l}\text { IAEA Nuclear Data Section, } \\
\text { Vienna, Austria }\end{array}$ & $\begin{array}{l}\text { IAEA Nuclear Data Section, } \\
\text { Vienna, Austria (Co-ordination) }\end{array}$ \\
\hline $\begin{array}{l}\text { OECD NEA Data Bank, } \\
\text { Paris, France }\end{array}$ & $\begin{array}{l}\text { U.S. National Nuclear Data Center, } \\
\text { Brookhaven, USA (Master database) }\end{array}$ \\
\hline $\begin{array}{l}\text { U.S. National Nuclear Data Center, } \\
\text { Brookhaven, USA }\end{array}$ & $\begin{array}{l}\text { 13 data evaluation centers } \\
\text { in USA, Russia, Netherlands, China, France, } \\
\text { Japan, Kuwait, Belgium, Canada, UK }\end{array}$ \\
\hline $\begin{array}{l}\text { Russian Nuclear Data Center, } \\
\text { Obninsk, Russia }\end{array}$ & $\begin{array}{l}\text { Data dissemination centers } \\
\text { (IAEA, OECD-NEA, USA, France, Sweden) }\end{array}$ \\
\hline $\begin{array}{l}\text { 9 co-operating Specialized centers } \\
\text { (Russia, China, Japan, Hungary, Korea, Ukraine) }\end{array}$ & \\
\hline
\end{tabular}

Table 2. Nuclear data center networks

\section{Overview of data libraries at IAEA}

The IAEA Nuclear Data Section holds a total of about 100 nuclear data libraries, representing enormous economic and scientific value. All libraries and the related documentation are available free of charge to scientists in IAEA member states. An overview is given in the document Index of $\mathrm{Nu}$ clear Data Libraries available from the IAEA Nuclear Data Section [8]. Brief descriptions of contents and/or format for most libraries are published in the IAEA-NDS- report series [9].

\section{II.1 General purpose libraries}

- Nuclear Wallet Cards [10]: Basic properties of ground and metastable states, available as pocket booklet (from US-NNDC) and online.

- NuDat [11]: User-friendly extract of most important data (for applications) from ENSDF, plus thermal neutron data (cross sections and resonance integrals). Available online.

- MIRD [12] - "Medical Internal Radiation Dose": Based on ENSDF, data processed with code "RADLST". Tables include intensities, energies and doses of all produced radiations, including X-rays, Auger electrons, etc., and decay scheme plots, for the selected nuclide. Available online.

- ENSDF [13] (Evaluated Nuclear Structure Data File) is the "master library" for structure and decay data, resulting from a continuous international evaluation effort coordinated by the IAEA. The master file is maintained by the US-NNDC, and contains evaluated experimental data for most known nuclides in the mass range 1 - 277. The evaluations are individually undertaken for mass chains (e.g. A=235) and are published in the journal Nuclear Data Sheets. Available online.

- NSR [14] (Nuclear Science References): Bibliographic database for low and intermediate energy nuclear physics. This is the main bibliography for structure and decay data and for non-neutron reaction data, and covers the literature from 1910 to present. Published regularly in the journal Nuclear Data Sheets (Recent References). Available online.

- CINDA [15] (Computer Index of Neutron Data): Bibliography of neutron data literature, covering also unpublished reports and computer files; also includes $(\gamma, \mathrm{n}),(\gamma, \mathrm{f})$ and spontaneous fission data. References describing the same experiment are listed together. An extension of the database to include chargedparticle induced and all photonuclear reactions is under preparation. Available online, as a book in several volumes, and on CD-ROM.

- EXFOR (called CSISRS in the USA): Experimental nuclear reaction data, compiled continuously by the network of nuclear reaction data centers which is coordinated by NDS. Contains neutron, charged-particle induced and photonuclear data, including integral and all types of differential cross sections, resonance parameters, polarization data, fission product yields, and many related data types. The library contains both numerical data tables and a structured abstract with experimental and bibliographic information. The neutron data in EXFOR are linked to the CINDA bibliography. The data can be retrieved in several formats, including computational format for further processing, and graphs. The main usage of EXFOR is for evaluators (EXFOR database is the starting point for 
all cross section evaluations), for applied users if no evaluation is available, and for researchers measuring or calculating cross section data. Available online and on CD-ROM [16].

- Evaluated cross section data libraries in ENDF-6 format [17]: the major "general purpose" evaluated libraries ENDF-B/VI (USA), BROND-2 (Russia), JEF2 (Europe), JENDL-3 (Japan), CENDL-2 (China) are available online ("ENDF" database) and on CD-ROM [18]. All of these libraries cover the neutron energy range from $10^{-5} \mathrm{eV}$ to $20 \mathrm{MeV}$, with some evaluations extending to higher energies (partly up to $1 \mathrm{GeV}$ ) and also including some charged-particle induced reactions. Output is available in several formats including plots, and software packages (Pre-Processing and Utility codes [19]) are available for data treatment. Since the original evaluations use resonance parameter representation, derived pointwise data are also available [20]. Online comparisons of evaluated and experimental cross sections can be done using the package ZVView [21] (see Fig. 1).

- Atomic Masses 1995 [22], a mass evaluation for more than 2900 nuclides. Available online.

\section{II.2 Selected special data libraries}

In addition to the general purpose libraries listed above, the IAEA Nuclear Data Section holds many specialized libraries and databases. A selection of important examples is briefly described below.

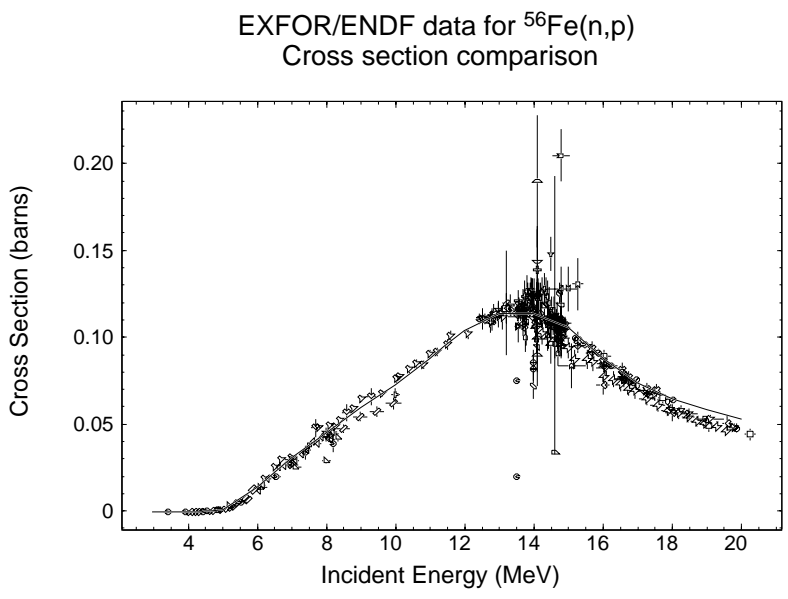

Figure 1. Online comparison of evaluated and experimental data using the package ZVView.

\section{II.2.1 Specialized libraries: IAEA products}

The following databases were developed or co-ordinated through IAEA projects.

- FENDL-2 [23]: Comprehensive cross section library for fusion and other applications, resulting from a worldwide effort including extensive testing, coordinated by IAEA. Consists of several sublibraries for transport calculations, activation, fusion reactions, etc. Available online and on CD-ROM.

- IAEA Photonuclear Data Library [24]: Evaluated photonuclear cross sections for 164 isotopes for energies up to $140 \mathrm{MeV}$ (most isotopes), up to about 25 $\mathrm{MeV}$ for others; for shielding, medical and other applications. Available online.

- Charged Particle Cross Section Database for Medical Radioisotope Production [25]: Production cross sections for $\gamma$-emitters, positron emitters and for monitor reactions, for incident protons through $\alpha$-particles up to about $30 \mathrm{MeV}$. Available online.

- RNAL [26] (Reference Neutron Activation Library): Evaluated cross sections for 255 neutron-induced reactions leading to radioactive products, for activation analysis and various other applications. The evaluations were extracted from various regional and national projects. Available online and on CD-ROM.

- RIPL [27] (Reference Input Parameter Library): Database and handbook for nuclear model calculations of nuclear reaction data, available online and on CD-ROM.

- NMF-90 [28] (Neutron Metrology File): Integrated database for neutron spectrum adjustment (unfolding) calculations. Available online or on diskettes.

- XG Standards [29] (X-ray and $\gamma$-ray standards for detector calibration): This database of selected nuclides contains their half-lives and the energies and emission probabilities of $\gamma$-rays and X-rays suitable for detector efficiency calibration. Available on diskette and online. An update is in preparation.

- NGATLAS [30]: Atlas of neutron capture cross sections for 739 targets from $10^{-5} \mathrm{eV}$ to $20 \mathrm{MeV}$. Available as a handbook (plots only) or online.

- SGNucDat [31] (Nuclear Data for Safeguards): PC database (also available online and as a handbook) containing nuclear data for the development and application of nuclear material accounting techniques.

\section{II.2.2 Examples of other specialized libraries available from IAEA}

- EPDL97 [32] (Evaluated Photon Data Library): Photon interaction data from $1 \mathrm{eV}$ to $100 \mathrm{GeV}$ from Lawrence Livermore National Laboratory, available on CD-ROM.

- Thermal Neutron Capture Gamma Rays [33]: Tables of energies and intensities of $\gamma$-rays produced in thermal neutron capture, by target or by $\gamma$-energy. Available online. 
- RRDF-98 [34] (Russian Reactor Dosimetry File): Neutron cross sections for 22 dosimetry reactions. Available online.

- MENDL-2 and MENDL-2P [35]: Activation cross sections for 505 stable and unstable targets between $\mathrm{Al}$ and Po for incident neutrons up to $100 \mathrm{MeV}$ and incident protons up to $200 \mathrm{MeV}$, for transmutation and other applications. Available by FTP.

- WIND and WIND-2 [36]: Cross sections for U, Np, and $\mathrm{Pu}$ isotopes for waste incineration, including neutron cross sections up to $100 \mathrm{MeV}$, proton cross sections for ${ }^{238} \mathrm{U}$, and neutron activation data for ${ }^{239} \mathrm{Pu}$ up to $2 \mathrm{GeV}$.

- "Maslov" library [37]: Evaluated neutron cross sections for $\mathrm{Np}, \mathrm{Pu}, \mathrm{Am}$ and $\mathrm{Cm}$ isotopes.

\section{Data access and services}

The main method of distributing numerical nuclear data in the early 21 st century is via the Internet, and therefore the IAEA Nuclear Data Section offers a variety of electronic services. At the same time, conventional mail services such as: sending customized retrievals or complete libraries on magnetic tape, CD-ROM, diskettes, hardcopy, or by e-mail, have been maintained for the convenience of users with varying needs and technical infrastructures. Users are kept up to date about new data libraries and other developments through the IAEA's Nuclear Data Newsletter [38].

\section{III.1 Online services}

- Worldwide Web (WWW): The IAEA Nuclear Data Services web page can be found at the web address (URL) http://www-nds.iaea.or.at (IAEA Vienna) or http://www-nds.ipen.br (IPEN) and contains interactive access to the major databases as well as an overview of all nuclear data libraries and databases available from the IAEA (IAEA Nuclear Data Guide), access to various reports, documents and manuals, nuclear data utility programs, the IAEA's Nuclear Data Newsletter, and a link to the Telnet-based nuclear data service NDIS.

- FTP: The IAEA Nuclear Data Section keeps several accounts for file transfer requiring no password (all accessible by the FTP addresses iaeand.iaea.or.at and $w w w$-nds.ipen.br, respectively): ANONYMOUS contains several complete libraries, utility codes and documents for public use; FENDL2 and RIPL permit access to the respective data libraries; NDSONL contains files saved by users of the Telnet-based online system "NDIS" (Nuclear Data Information System) to "local area"; NDSOPEN is used for bilateral file exchange.
- TELNET service ("NDIS"): The starting command is telnet iaeand.iaea.org or telnet www-nds.ipen.br, then the username iaeands has to be entered. A detailed manual is available online in PostScript or as a hardcopy [39]. This type of online service has lost importance due to the rapidly expanding WWW technology but may still be useful in certain situations. NDIS provides access to the main interactive nuclear databases (CINDA, EXFOR, ENSDF, NUDAT, NSR, ENDF) as well as to PostScript documents and utility programs.

\section{III.2 Computer programs}

Under most circumstances, nuclear data related computer codes are distributed by the OECD NEA Data Bank [40] in Paris, France to scientists from IAEA member states, or, for codes of US origin, by the Radiation Shielding Information Computational Center (RSICC) [41], Oak Ridge, USA (a handling fee may be charged). However, some utility programs and PC packages are available directly from NDS, online, by FTP or on CD-ROM.

\section{III.3 Documents}

Hardcopy documents published by NDS include handbooks, research and meeting reports (INDC report series), data library documentations (IAEA-NDS report series), and the Nuclear Data Newsletter. Most new reports are also made available electronically on the WWW in PDF format. NDS can be contacted by e-mail under services@iaeand.iaea.org to request hardcopy documents, other mail services, or other nuclear data related information.

\section{III.4 Trends}

Improvement of the nuclear data services has started on a network-wide basis. To make the service more user-friendly and, at the same time, extend the retrieval possibilities, development of a combined nuclear reaction database is under way, using advanced database software, with which the EXFOR, CINDA and ENDF libraries will appear as one database to the user. To improve services for users without good Internet connections to the IAEA, the NDS is pursuing two further developments: distribution of databases on CD-ROM, potentially with a web-type user interface and the possibility of automatic updates through the Internet; and the creation of additional regional copies of the "Nuclear Data Services" website ("mirror sites").

\section{References}

[1] D.D. Sood, P. Obložinský, M. Herman and O. Schwerer, Nuclear Data for Applications, J. Radioanal. Nucl. Chem. 243, 227 (2000)

[2] See http://www-amdis.iaea.org/ 
[3] R.B. Firestone, V.S. Shirley, S.Y.F. Chu, C.M. Baglin, and J. Zipkin, (ed.), Table of Isotopes, 8th edition (1996), John Wiley and Sons, New York

[4] S.Y.F. Chu, H. Nordberg, R.B. Firestone and L.P. Ekström, Isotope Explorer, see http://ie.lbl.gov/isoexpl/isoexpl.htm

[5] G. Audi, O. Bersillon, O., J. Blachot and A.H. Wapstra, The NUBASE evaluation of nuclear and decay properties, Nucl. Phys. A624, 1-124 (1997). The database is available in electronic form from the Atomic Mass Data Center, see http://csnwww.in2p3.fr/amdc/.

[6] V.G. Pronyaev (ed.), The network of nuclear reaction data centers, Report INDC(NDS)-401, Rev.3, July 2000 (IAEA, Vienna, Austria)

[7] V.G. Pronyaev (ed.), Nuclear Structure and Decay Data (NSDD) Network, Report INDC(NDS)-421, February 2001 (IAEA, Vienna, Austria)

[8] O. Schwerer, H.D. Lemmel, (ed.), Index of Nuclear Data Libraries available from the IAEA Nuclear Data Section, Report IAEA-NDS-7, July 2002 (IAEA, Vienna, Austria). See also http://www-nds.iaea.or.at/reports/nds-7.pdf

[9] H.D. Lemmel, O. Schwerer, (ed.), Index to the IAEANDS Documentation Series, Report IAEA-NDS-0, July 2002 (IAEA, Vienna, Austria). See also http://wwwnds.iaea.or.at/nds-0.html

[10] J.K. Tuli (ed.), Nuclear Wallet Cards, Sixth edition, January 2000 (National Nuclear Data Center, Brookhaven, USA)

[11] C.L. Dunford and T.W. Burrows, NuDat System for Access to Nuclear Data, Report IAEA-NDS-205 (Revision 98/7), July 1998 (IAEA, Vienna, Austria)

[12] MIRD - Medical Internal Radiation Dose, available through http://www-nds.iaea.or.at/formmird.html.

[13] See http://www-nds.iaea.or.at/ensdf/

[14] See http://www-nds.iaea.or.at/nsr/

[15] CINDA 2000, The Index to Literature and Computer Files on Microscopic Neutron Data (IAEA, Vienna, Austria, November 2000)

[16] See http://www-nds.iaea.or.at/exfor/. For an introduction, see V. McLane, EXFOR Basics: A Short Guide to the Nuclear Reaction Data Exchange Format, Report BNL-NCS-63380, BNL, Brookhaven, 1999

[17] V. McLane (ed.), ENDF-102 Data Formats and Procedures for the Evaluated Nuclear Data File ENDF-6, Report BNLNCS-44945-01/04-Rev., April 2001

[18] H.D. Lemmel, Index to BROND-2, CENDL-2, ENDF/B-6, $J E F-2, J E N D L-3, I R D F, E F F-2.4$ and FENDL/E, Report IAEA-NDS-107 (Revision 11), March 1996 (IAEA, Vienna, Austria)

[19] See http://www-nds.iaea.or.at/ndspub/endf/prepro/ and http://www-nds.iaea.or.at/ndspub/endf/utility/

[20] See $h t t p: / / w w w-n d s . i a e a . o r . a t / e n d f / e n d f f r a m e . h t m l$ for pointwise cross sections at $300 \mathrm{~K}$ temperature from several main libraries. In addition, the database "point2000" CONTAINS POINTWISE CROSS SECTIONS FROM endf/b-vi AT 8 TEMPERATURES, SEE http://wwwnds.iaea.or.at/point2000/
[21] V. Zerkin, ZVView: Graphic Software for Nuclear Data Analysis, see http://www-nds.iaea.or.at/ndspub/zvview/

[22] G. Audi and A.H. Wapstra, The 1995 Update to the Atomic Mass Evaluation, Nucl. Phys. A595, 409-480 (1995). See also Report IAEA-NDS-47 (Revision 3), November 1995 (IAEA, Vienna, Austria)

[23] A.B. Pashchenko, H. Wienke and D.W. Muir, FENDL-2: An improved nuclear data library for fusion applications, in: Proceedings of International Conference on Nuclear Data for Science and Technology, Trieste, Italy, 19-24 May 1997, ed. by G. Reffo, A. Ventura and C. Grandi, (Italian Physical Society, Conference Proceedings Vol. 59, Bologna, 1998) pp. 1150 - 1154

[24] Handbook on Photonuclear Data for Applications, IAEATECDOC-1178, October 2000 (IAEA, Vienna, Austria)

[25] Charged-Particle Cross Section Database for Medical Radioisotope Production, IAEA-TECDOC-1211, May 2001 (IAEA, Vienna, Austria)

[26] See http://www-nds.iaea.or.at/ndspub/rnal/www/

[27] Handbook for Calculations of Nuclear Reaction Data (Reference Input Parameter Library), IAEA-TECDOC-1034, August 1998 (IAEA, Vienna, Austria). See also http://wwwnds.iaea.or.at/ripl and CD-ROM (IAEA-NDS-CD-02, Vienna, May 1998)

[28] E.M. Zsolnay, E.J. Szöndi and H.J. Nolthenius, The Neutron Metrology File NMF-90, Report IAEA-NDS-171, Rev. 1, January 1999 (IAEA, Vienna, Austria)

[29] X -ray and gamma-ray standards for detector calibration, IAEA-TECDOC-619, September 1991 (IAEA, Vienna, Austria). Data available on the WWW through http://www.nucleartraining.co.uk/iaea/index.htm

[30] Atlas of Neutron Capture Cross Sections, ed. by J. Kopecky, graphs published in Report INDC(NDS)-362, April 1997 (IAEA, Vienna, Austria). Numerical data as well as plots are available online through http://www-nds.iaea.or.at/ngatlas2/

[31] N. Kocherov, M. Lammer and O. Schwerer, Handbook of Nuclear Data for Safeguards, Report INDC(NDS)-376, December 1997 (IAEA, Vienna, Austria). See also http://wwwnds.iaea.or.at/sgnucdat/sgnuc.html

[32] EPDL97 - The Evaluated Photon Data Library, '97 version, by D.E. Cullen, J.H. Hubbell and L. Kissel, LLNL (USA). For summary documentation of contents see V.P. Pronyaev and P.K. McLaughlin (ed.), Report IAEA-NDS-196, August 1998 (IAEA, Vienna, Austria)

[33] Thermal Neutron Capture Gamma Rays, available through http://www-nds.iaea.or.at/wallet/tnc/capgam.shtml

[34] K.I. Zolotarev, A.V. Ignatyuk, V.N. Manokhin and A.B. Pashchenko, RRDF-98. Russian Reactor Dosimetry File, Report IAEA - NDS - 193, March 1999 (IAEA, Vienna, Austria). Data available online under http://www-nds.iaea.or.at/reports/nds-193.htm

[35] MENDL-2, Neutron reaction data library for nuclear activation and transmutation at intermediate energies, by Yu.N. Shubin et al., Summary documentation by H.D. Lemmel, Report IAEA-NDS-136 (Revision 1), July 1997 (IAEA, Vienna, Austria), and MENDL-2P, Proton reaction data library for nuclear activation by Yu.N. Shubin et al., Report IAEANDS-204, January 1998 (IAEA, Vienna, Austria) 
[36] A.Yu. Konobeyev et al., WIND, Evaluated neutron crosssection data up to $100 \mathrm{MeV}$ for incineration of actinides. With one data file of proton-induced reactions for U-238. Report IAEA-NDS-143, March 1995 (IAEA, Vienna, Austria)

[37] V. Maslov et al., Evaluated neutron reaction data for $N p$, $\mathrm{Am}, \mathrm{Cm}$ and Pu isotopes. Summary documentation: Report IAEA-NDS-164 (Revision 3), February 1998 (IAEA, Vienna, Austria)

[38] Nuclear Data Newsletter (IAEA, Vienna, Austria). Is- sued 2 times per year. Also available through http://wwwnds.iaea.or.at/newslett.html

[39] C.L. Dunford and T.W. Burrows, Online Nuclear Data Service, Report IAEA-NDS-150 (Revision 99/3), March 1999 (IAEA, Vienna, Austria)

[40] See http://www.nea.fr/html/dbprog/

[41] See http://www-rsicc.ornl.gov/rsic.html 